\begin{tabular}{|c|l|}
\hline Title & Discrimination of quantum dots using an optically created nuclear field \\
\hline Author(s) & Sasakura, H.; Kaji, R.; A dachi, S.; Muto, S. \\
\hline Citation & $\begin{array}{l}\text { A pplied Physics Letters, 92(4), 041915-1-041915-3 } \\
\text { https://doi.org/10.1063/1.2839615 }\end{array}$ \\
\hline Issue Date & 2008 \\
\hline Doc URL & http://hdl.handle.net/2115/49865 \\
\hline Type & article \\
\hline File Information & GetPDFServlet.pdf \\
\hline
\end{tabular}

Instructions for use 


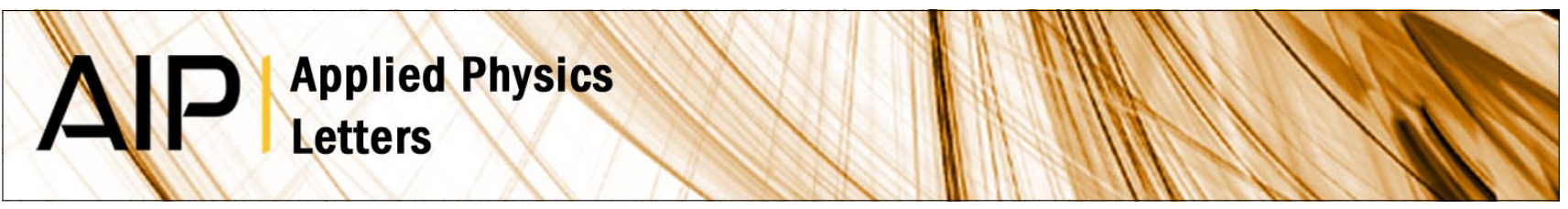

\section{Discrimination of quantum dots using an optically created nuclear field}

H. Sasakura, R. Kaji, S. Adachi, and S. Muto

Citation: Appl. Phys. Lett. 92, 041915 (2008); doi: 10.1063/1.2839615

View online: http://dx.doi.org/10.1063/1.2839615

View Table of Contents: http://apl.aip.org/resource/1/APPLAB/v92/i4

Published by the American Institute of Physics.

\section{Related Articles}

Enhancement of spontaneous emission in three-dimensional low refractive-index photonic crystals with designed defects

Appl. Phys. Lett. 101, 071109 (2012)

Optical properties and effect of carrier tunnelling in CdSe colloidal quantum dots: A comparative study with different ligands

AlP Advances 2, 032132 (2012)

Controlling the photoluminescence of acceptor and donor quantum dots embedded in a nonlinear photonic crystal

Appl. Phys. Lett. 101, 051115 (2012)

Nanoscale fluorescence imaging with quantum dot near-field electroluminescence

Appl. Phys. Lett. 101, 043118 (2012)

Theoretical optimization of multi-layer InAs/GaAs quantum dots subject to post-growth thermal annealing for tailoring the photoluminescence emission beyond $1.3 \mu \mathrm{m}$

J. Appl. Phys. 112, 024315 (2012)

\section{Additional information on Appl. Phys. Lett.}

Journal Homepage: http://apl.aip.org/

Journal Information: http://apl.aip.org/about/about_the_journal

Top downloads: http://apl.aip.org/features/most_downloaded

Information for Authors: http://apl.aip.org/authors

\section{ADVERTISEMENT}

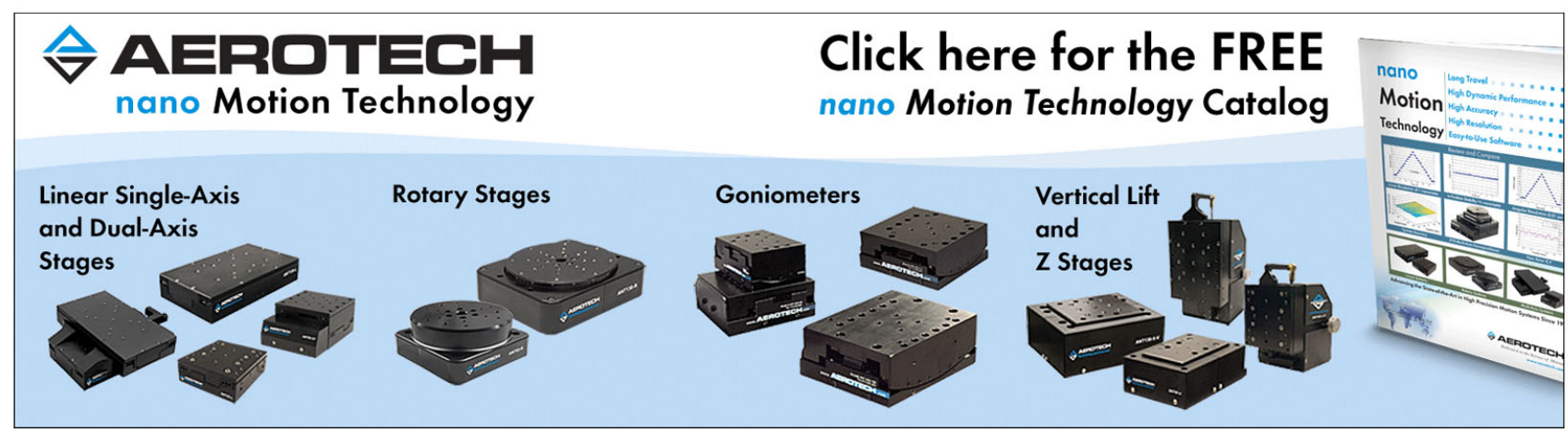




\title{
Discrimination of quantum dots using an optically created nuclear field
}

\author{
H. Sasakura ${ }^{a)}$ \\ CREST, Japan Science and Technology Agency, Kawaguchi 332-0012, Japan \\ R. Kaji, S. Adachi, ${ }^{\text {b),c) }}$ and S. Muto ${ }^{\text {c) }}$ \\ Department of Applied Physics, Hokkaido University, Sapporo 060-8628, Japan
}

(Received 4 December 2007; accepted 14 January 2008; published online 1 February 2008)

\begin{abstract}
We investigated an optically created nuclear field in a single InAlAs quantum dot and demonstrated that the nuclear field can be used to discriminate whether photoluminescence lines originate from the same dot or a different dot. Since the nonlinear response of the nuclear field is sensitive to the electron $g$ factor and correlation time of a coupled electron-nuclear spin system, the resultant Overhauser shift is their good measure for individual quantum dots. This method provides a simple and convenient alternative to the standard photon cross-correlation method. (C) 2008 American Institute of Physics. [DOI: 10.1063/1.2839615]
\end{abstract}

Single quantum dot (QD) devices attract considerable attention in the field of semiconductor nanostructures because of their potential for single photon emission, single electron storage, and manipulation of single qubits for quantum information processing. ${ }^{1}$ Therefore, single QD spectroscopy plays an increasingly important role of exploring and controlling these properties. In general, many photoluminescence (PL) lines are observed even for a truly single QD since exciton complexes such as neutral biexciton, positively, and negatively charged excitons are generated under the usual excitation conditions. Further, when several QDs exist in the measurement area, e.g., in a single mesa structure, PL lines of the exciton families from other QDs also emerge. In the standard spectroscopic procedure, we need to assign the origins of the observed PL lines. By various measurements for the assignments, ${ }^{2}$ it is possible to clarify the origin of an individual PL line; however, the procedure to achieve this is cumbersome. Primarily, it is necessary to discriminate whether the emissions originate from the same single QD or a different QD. This can be achieved by a photon crosscorrelation measurement using a Hanbury-Brown and Twiss (HBT) $\operatorname{setup}^{3}$ or by single QD selection from a microimage of the emissions using a pinhole. ${ }^{4}$ These methods are very effective, however, HBT measurement generally requires care and takes a long time because of large photon loss, and QD selection through a pinhole is effective only in case of single-stacked QDs.

Recently, research on electron-nuclear spin interaction has been revived considering its applications. This is because semiconductor QDs enhance the electron-nuclear spin exchange interaction (hyperfine interaction) due to the strong three-dimensional confinement of the electron wavefunction, and the enhanced interaction allows the possibility of aligning nuclear spins in one direction up to several tens of percentage in a single QD through optical pumping. In fact, a high rate of nuclear spin polarization and the resultant large effective nuclear field up to several tesla were recently ob-

\footnotetext{
${ }^{a)}$ Present address: Research Institute of Electronic Science, Hokkaido University, Sapporo 001-0021, Japan. Electronic mail: hirotaka@ eng.hokudai.ac.jp.

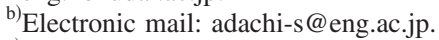

${ }^{c}$ Also at CREST, Japan Science and Technology Agency, Kawaguchi 332-0012, Japan.
}

served in interface GaAs QDs, ${ }^{5,6}$ self-assembled InAlAs QDs, ${ }^{7-9}$ and InGaAs QDs. ${ }^{10-12}$ Because of the ultralong coherence, nuclear spin is expected to contribute to applications such as a long-lived quantum memory at the nuclear level $^{13}$ and qubit conversion using a nuclear field. ${ }^{14}$ Apart from such potential applications for quantum information processing, the nuclear field is also useful for the evaluation of the magnitude and sign of an electron and hole $g$ factors, ${ }^{15}$ which is based on the fact that a nuclear field affects only electrons and can compensate for the external magnetic field. In this study, we demonstrate a simple discrimination method of PL originating from individual QDs. This method is based on the sensitivity of the optically created nuclear field for individual QDs to the electron $g$ factor, the electron spin coherence, etc.

The sample used here was $\operatorname{In}_{0.75} \mathrm{Al}_{0.25} \mathrm{As} \mathrm{QDs}$ grown on a $\mathrm{Al}_{0.3} \mathrm{Ga}_{0.7} \mathrm{As} / \mathrm{GaAs}$ buffer layer on an undoped (100) GaAs substrate by molecular beam epitaxy: ${ }^{16}$ density $\sim 5$ $\times 10^{10} \mathrm{~cm}^{-2}$, diameter $\sim 20 \mathrm{~nm}$, and height $\sim 3 \mathrm{~nm}$. A small mesa structure with a typical top lateral diameter of $\sim 150 \mathrm{~nm}$ was fabricated to limit the observable QDs. On the average, there are several QDs in a mesa; however, some mesas only have one or a few QDs due to inhomogeneous QD distribution, in which well-separated sharp QD emissions are observed. The micro-PL measurements were performed at $5 \mathrm{~K}$ under the longitudinal external magnetic field $B_{z}$ up to $5 \mathrm{~T}$ in a Faraday configuration. A cw-Ti:sapphire laser beam traveling along the QD growth direction was focused on the sample surface using a microscope objective. The QD emissions collected by the same objective lens were dispersed using a triple grating spectrometer and detected using a liquid $\mathrm{N}_{2}$-cooled Si-charge-coupled device (CCD) camera. The spectral resolution that determines the resonant peak energies was $\sim 5 \mu \mathrm{eV}$, measured by the spectral fitting. The typical exposure time of the CCD camera to obtain one spectrum with a high signal-to-noise ratio was $1 \mathrm{~s}$.

Figure 1(a) shows the PL spectra of the InAlAs QDs in a single mesa structure at $5 \mathrm{~K}$ and $0 \mathrm{~T}$. The sample was excited at the wetting layer $(\sim 1.698 \mathrm{eV})$ by a linearly polarized light. The five PL peaks labeled as P1-P5 are observed clearly in this energy region. Figure 1(b) shows the application of $B_{z}$ results in the Zeeman splitting of each peak with a blueshift due to a diamagnetic effect. Figure 1(b) also shows 
(a)

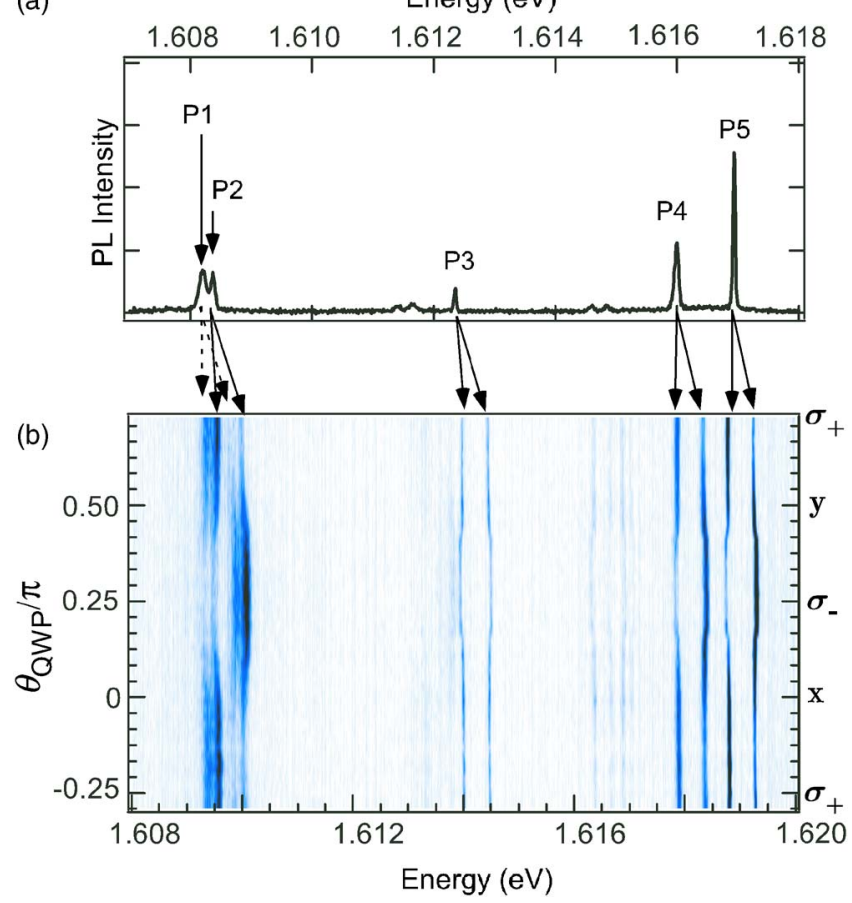

FIG. 1. (Color online) (a) PL spectra recorded at $B_{z}=0 \mathrm{~T}$ for an excitation wavelength of $\sim 730 \mathrm{~nm}$. (b) A CCD image of the Zeeman-split PL lines at $B_{z}=4 \mathrm{~T}$ as a function of the rotating angle of QWP $\left(\theta_{\mathrm{QWP}}\right)$ that was inserted in an excitation path. $\theta_{\mathrm{QWP}}=-0.25 \pi, 0,0.25 \pi$, and $0.5 \pi$ correspond to the $\sigma_{+}, x, \sigma_{-}$, and $y$ polarized excitations, respectively.

the contour plot of the PL intensity at $B_{z}=4 \mathrm{~T}$ as a function of the degree of circular polarization of the excitation light. The PL spectra were obtained by rotating the angle $\theta_{\mathrm{QWP}}$ of a quarter-wave plate (QWP) inserted in the excitation beam path. $\theta_{\mathrm{QWP}}=-0.25 \pi, 0,+0.25 \pi$, and $+0.5 \pi$ correspond to the $\sigma_{+}, x, \sigma_{-}$, and $y$ polarized excitations, respectively. The difference in Zeeman energy is observed with linearly polarized excitation $\left(\theta_{\mathrm{QWP}}=0\right.$ and $\left.0.5 \pi\right)$; the values $\sim 483 \mu \mathrm{eV}$ ( $\mathrm{P} 1$ and $\mathrm{P} 2$ ) and $\sim 466 \mu \mathrm{eV}$ (P3-P5) indicate that P1-P5 can be divided into two groups: group I (P1 and P2) and group II (P3-P5). In fact, by various measurements for the assignments, the PLs of each group originate from different QDs and those are caused by the emissions of positively charged excitons (P2 and P5), neutral excitons (P1 and P4), and neutral biexciton (P3). ${ }^{17}$ In addition, the figure clearly indicates that the Zeeman splitting differs depending on the excitation polarization and shows a similar dependence for all PL peaks; larger Zeeman splittings appear around $\theta_{\mathrm{QWP}}=$ $+0.25 \pi$ despite a constant $B_{z}$. The change in the splitting energy from that with linearly polarized excitation is known as the Overhauser shift (OHS) ${ }^{18}$ OHS is expressed as $A\left\langle I_{z}\right\rangle$ $\times\left(=g_{z}^{e} \mu_{B} B_{N}\right)$ by the averaged nuclear spin polarizations $\left\langle I_{z}\right\rangle$ and the resultant nuclear field $B_{N}$, where $A, g_{z}^{e}$, and $\mu_{B}$ are the averaged hyperfine constant, electron $g$ factor in the QD growth direction, and Bohr magneton $(\sim 58 \mu \mathrm{eV} / \mathrm{T})$. Therefore, OHS is a good indicator of $\left\langle I_{z}\right\rangle$ and $B_{N}$ in a single QD. Close observation of the spectra reveals that the asymmetrical OHS between $\sigma_{+}$and $\sigma_{-}$excitations can be detected for all PLs, as shown in Fig. 2.

The steady state $\left\langle I_{z}\right\rangle$ is determined by the balance between the nuclear spin polarization and its depolarization, as shown in the following rate equation: ${ }^{19,20}$

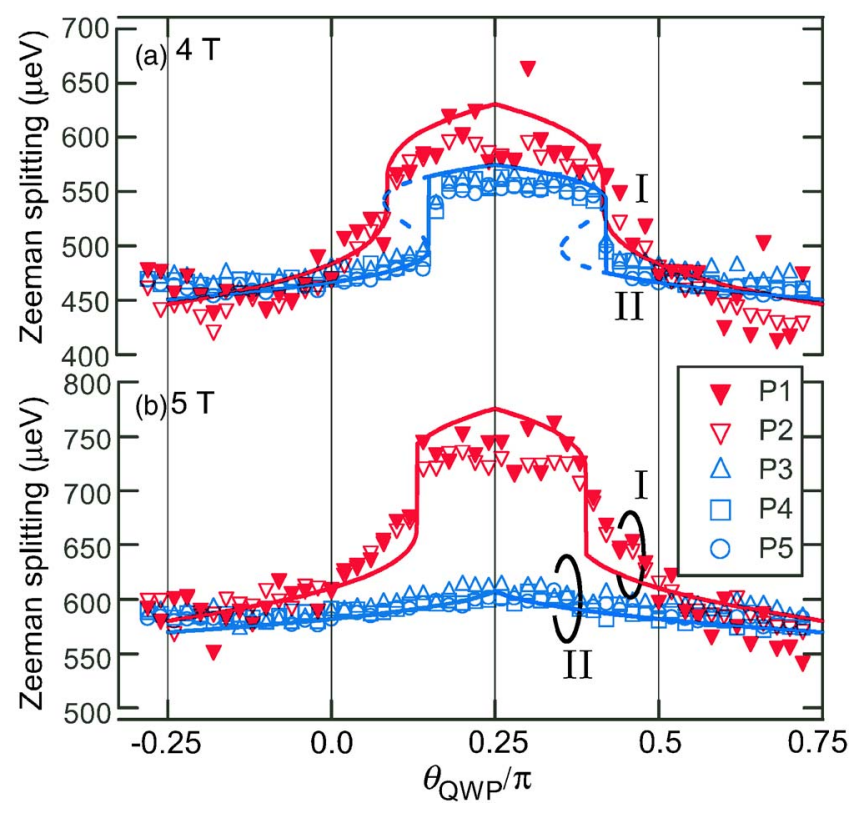

FIG. 2. (Color online) [(a) and (b)] Zeeman splitting of P1-P5 as a function of $\theta_{\mathrm{QWP}}$ at $B_{z}=4$ and $5 \mathrm{~T}$, respectively. Under the same excitation conditions, the OHSs are clearly different between the groups I (P1 and P2) and II (P3-P5). Solid curves are calculated by Eqs. (1) and (2) using $g_{z}^{e(\mathrm{I})}=-0.42, g_{z}^{e(\mathrm{II})}=-0.34, \tau_{c}^{(\mathrm{I})}=18.8 \mathrm{ps}, \tau_{c}^{(\mathrm{II})}=38.7 \mathrm{ps}, f=0.2, N^{(\mathrm{II})}=28000$, $N^{(I I)}=44000$, and $A=52.6 \mu \mathrm{eV}$. Dashed curve indicates the hysteresis behavior in the $\left\langle S_{z}\right\rangle$ dependence of the Overhauser shift calculated with the same parameters of group II.

$$
d\left\langle I_{z}\right\rangle / d t=\left[Q\left(\left\langle S_{z}\right\rangle-S_{0}\right)-\left\langle I_{z}\right\rangle\right] / T_{\mathrm{NF}}-\left\langle I_{z}\right\rangle / T_{\mathrm{ND}},
$$

where $\left\langle S_{z}\right\rangle$ is the averaged electron spin polarization, $S_{0}$ is the thermal electron spin polarization, $1 / T_{\mathrm{NF}}$ and $1 / T_{\mathrm{ND}}$ are the nuclear spin polarization and depolarization rates, respectively, and $Q(=[I(I+1)] /[S(S+1)])$ is a momentum conversion coefficient from electron spin to nuclear spin system in the spin flip-flop process. Based on the general form of the spin-flip process of the precessional decoherence type, ${ }^{19}$ the spin transfer rate $1 / T_{\mathrm{NF}}$ is expressed as follows by assuming uniform electron wavefunction in a $\mathrm{QD}::^{10-12}$

$$
\frac{1}{T_{\mathrm{NF}}}=\tau_{c} f\left(\frac{A}{N \hbar}\right)^{2} /\left[1+\left(\frac{\tau_{c}}{\hbar}\right)^{2}\left\{g_{z}^{e} \mu_{B}\left(B_{z} \pm B_{N}\right)\right\}^{2}\right],
$$

where $\tau_{c}, N$, and $f$ indicate the correlation time of the coupled electron-nuclei system with the broadening $\hbar / \tau_{c}$, number of nuclei in a QD, and electron existence probability in a $\mathrm{QD}$, respectively. The magnitude of $B_{N}$ depends on the steady state $\left\langle I_{z}\right\rangle$, as $B_{N}=A\left\langle I_{z}\right\rangle / g_{z}^{e} \mu_{B}$, and the direction can be selected as parallel or antiparallel to $B_{z}$ according to the helicity of circular excitation. According to Eq. (2), the compensation of $B_{z}$ by $B_{N}$ reduces the electron Zeeman splitting, i.e., $g_{z}^{e} \mu_{B}\left(B_{z}-B_{N}\right)$ and results in the increase of $1 / T_{\mathrm{NF}}$. Note that the $B_{N}$ affects only the electron due to the nonzero probability at the nucleus site. ${ }^{19}$

Figure 2 shows the Zeeman splitting of P1-P5 plotted as a function of $\theta_{\text {QwP. }}$. The Zeeman splitting energies were obtained by Lorentzian fitting of all PL spectra, for example, in Fig. 1(b) for $B_{z}=4 \mathrm{~T}$. In the figure, the energy difference from that at $\theta_{\mathrm{QWP}}=0(0.5 \pi)$ corresponds to the OHS. At $B_{z}=4 \mathrm{~T}$, the Zeeman splittings of all PL lines show a positive abrupt change of OHS around $\theta_{\mathrm{QWP}}=0.25 \pi$. This abrupt change, so-called "nuclear spin switching," occurs because of the bistable behavior of the steady state $\left\langle I_{z}\right\rangle$ on the exci- 
tation polarization and power. ${ }^{11}$ The significantly asymmetrical OHS and the abrupt change of OHS prove the validity of the above-mentioned formation mechanism of the nuclear spin polarizations: the explosive increase of the spin transfer rate of Eq. (2) for $B_{z}-B_{N} \sim 0$. Further, the positive abrupt change of OHS clearly shows that the electron and hole $g$ factors have opposite signs, $g_{z}^{e} \cdot g_{z}^{h}<0$. If $g_{z}^{e} \cdot g_{z}^{h}>0$, the observed total Zeeman splitting is the sum of the electron and hole Zeeman splitting and, therefore, it decreases due to the reduced electron Zeeman splitting when $B_{z}$ is compensated by $B_{N}$, as in the case of $\operatorname{In}(\mathrm{Ga})$ As QDs. In fact, we evaluated $g_{z}^{h(\mathrm{I})}=+2.52 \pm 0.01$ and $g_{z}^{e(\mathrm{I})}=-0.42 \pm 0.02$ for group I and $g_{z}^{h(\mathrm{II})}=+2.36 \pm 0.01$ and $g_{z}^{e(\mathrm{II})}=-0.34 \pm 0.02$ for group II by canceling $B_{z}$ with $B_{N}$ at one edge of the nuclear spin bistability. ${ }^{15}$

From the slope of the change around $\theta_{\mathrm{QWP}}=0.15 \pi$ and the maximum OHS around $\theta_{\mathrm{QWP}}=0.25 \pi$, groups I and II are found to be affected by the different nuclear fields. Considering the difference of the electron $g$ factor, the maximum $B_{N}$ 's observed in two groups are deduced: $B_{N}^{(\mathrm{I})} \sim-4.88 \mathrm{~T}$ and $B_{N}^{(\mathrm{II})} \sim-4.66 \mathrm{~T}$. Besides, the short correlation time induces a slow change of the nuclear field because $\hbar / \tau_{c}$ hinders the explosive increase of the spin transfer rate of Eq. (2) at $B_{z}$ $-B_{N} \sim 0$. Therefore, the observed slope of the change around $\theta_{\mathrm{QWP}}=0.15 \pi$ is affected by the difference of $\tau_{c}$ for the two groups. In fact, the spectral width of group I is larger than that of group II: P2 $(1.60836 \mathrm{eV}, 70 \mu \mathrm{eV})$ and P5 $(1.61694 \mathrm{eV}, 34 \mu \mathrm{eV})$. At $B_{z}=5 \mathrm{~T}$, the difference between the two groups stands out; the abrupt change of OHS disappears for group II, while group I still shows the asymmetric OHS. This difference can be explained by the change of the critical value of $B_{z}$ for the bistable behavior in the $B_{z}$-dependence. ${ }^{15}$ The critical value of $B_{z}$ for the abrupt change is sensitive to the QD properties; $g_{z}^{e}$ and $\tau_{c}$ under the same $B_{z}$. Therefore, above QD properties mainly determine the presence or absence of the abrupt change of OHS.

To confirm the above scenario, we reproduce the $\left\langle S_{z}\right\rangle$-dependence of OHS compared with the experimental results using Eqs. (1) and (2). The sweep direction of $\left\langle S_{z}\right\rangle$ from $-0.5\left(\theta_{\mathrm{QWP}}=-0.25 \pi\right)$ to $-0.5(0.75 \pi)$ via $0.5(0.25 \pi)$ is associated with the measurement conditions. In particular, the OHS of group II at $B_{z}=4 \mathrm{~T}$ shows a clear hysteresis behavior in the $\left\langle S_{z}\right\rangle$-dependence, that is, the OHS exhibits sweep direction dependence of $\left\langle S_{z}\right\rangle$ from $-0.5\left(\theta_{\mathrm{QWP}}=-0.25 \pi\right)$ to 0.5 $(0.25 \pi)$ and $0.5(0.25 \pi)$ to $-0.5(0.75 \pi)$. This hysteresis in the $\left\langle S_{z}\right\rangle$ dependence has already been observed in the previous studies ${ }^{9,10}$ and is consistent with the physics of nuclear spin switching that has been recently reported. ${ }^{11}$ We show the calculation curves of OHS in Fig. 2 considering the hysteresis behavior. Assuming that $\tau_{c}$ is deduced by the coherence time of electron spin, we evaluated $\tau_{c}^{(\mathrm{I})} \sim 18.8 \mathrm{ps}$ for group I and $\tau_{c}^{\text {(II) }} \sim 38.7 \mathrm{ps}$ for group II by the spectra widths of $\mathrm{P} 2$ and $\mathrm{P} 5$, which are assigned to positively charged excitons. These values are consistent with the observed correlation time obtained by Fourier spectroscopy. ${ }^{17}$ The following parameters are used for reproduction of the experimental results: $A \sim 52.6 \mu \mathrm{eV}, f \sim 0.2,{ }^{21} T_{\mathrm{ND}} \sim 50 \mathrm{~ms}, I(I+1)=12.25$, $N^{(\mathrm{I})}=28000$, and $N^{(\mathrm{II})}=44000$, where $A$ and $I(I+1)$ are weighted values for an $\mathrm{In}_{0.75} \mathrm{Al}_{0.25} \mathrm{As}$ QD. ${ }^{22}$ Although the dif- ference of $g_{z}^{e}$ and $\tau_{c}$ between the two groups is expected to be negligible because the differences in the emission energies are small $(\sim 8 \mathrm{meV})$, we observed great divergence in the nuclear field formation, implying that the shape and composition and strain of each QD are unique. The sensitivity of QD properties to the critical value of $B_{z}$ for the abrupt change of OHS facilitates a solution to discriminate the individual QDs.

We investigated the nuclear spin switching in InAlAs QDs and compared the behaviors in two different single QDs. The observed threshold value for the nuclear spin switching is found to be sensitive to the QD properties and, therefore, the switching is useful to discriminate the individual QDs in the same measurement area. This method provides a simple and convenient alternative to the standard photon cross-correlation method.

This work was financially supported by a Grant-in-Aid for Scientific Research of the Ministry of Education, Culture, Sports, Science, and Technology, Japan.

${ }^{1}$ D. D. Awschalom, N. Samarth, and D. Loss, Semiconductor Spintronics and Quantum Computation (Springer, Berlin, 2002).

${ }^{2}$ For example, H. Kumano, S. Kimura, M. Endo, H. Sasakura, S. Adachi, S. Muto, and I. Suemune, J. Nanoelectron. Optoelectron. 1, 39 (2006).

${ }^{3}$ R. Hanbury-Brown and R. Q. Twiss, Nature (London) 177, 27 (1956).

${ }^{4}$ L. Landin, M. S. Miller, M.-E. Pistol, C. E. Pryor, and L. Samuelson, Science 280, 262 (1998).

${ }^{5}$ D. Gammon, Al. L. Efros, T. A. Kennedy, M. Rosen, D. S. Katzer, D. Park, S. W. Brown, V. L. Korenev, and I. A. Merkulov, Phys. Rev. Lett. 86, 5176 (2001).

${ }^{6}$ A. S. Bracker, E. A. Stinaff, D. Gammon, M. E. Ware, J. G. Tischler, A. Shabaev, Al. K. Efros, D. Park, D. Gershoni, V. L. Korenev, and I. A. Merkulov, Phys. Rev. Lett. 94, 047402 (2005).

${ }^{7}$ T. Yokoi, S. Adachi, H. Sasakura, S. Muto, H. Z. Song, T. Usuki, and S. Hirose, Phys. Rev. B 71, 041307 (2005).

${ }^{8}$ T. Yokoi, S. Adachi, H. Sasakura, S. Muto, H. Z. Song, T. Usuki, and S. Hirose, Physica E (Amsterdam) 29, 510 (2005).

${ }^{9}$ R. Kaji, S. Adachi, H. Sasakura, and S. Muto, e-print arXiv:cond-mat/ 07091382.

${ }^{10}$ P.-F. Braun, B. Urbaszek, T. Amand, X. Marie, O. Krebs, B. Eble, A. Lemaitre, and P. Voisin, Phys. Rev. B 74, 245306 (2006).

${ }^{11}$ A. I. Tartakovskii, T. Wright, A. Russell, A. B. Van'kov, J. Skiba-Szymanska, I. Drouzas, R. S. Kolodka, M. S. Skolnick, P. W. Fry, A. Tahraoui, H.-Y. Liu, and M. Hopkinson, Phys. Rev. Lett. 98, 026806 (2007).

${ }^{12}$ P. Maletinsky, C. W. Lai, A. Badolato, and A. Imamoglu, Phys. Rev. B 75, 035409 (2007).

${ }^{13}$ J. M. Taylor, C. M. Marcus, and M. D. Lukin, Phys. Rev. Lett. 90, 206803 (2003).

${ }^{14}$ S. Muto, S. Adachi, T. Yokoi, H. Sasakura, and I. Suemune, Appl. Phys. Lett. 87, 112506 (2005).

${ }^{15}$ R. Kaji, S. Adachi, H. Sasakura, and S. Muto, Appl. Phys. Lett. 91, 261904 (2007).

${ }^{16}$ H. Sasakura, S. Adachi, S. Muto, H. Song, T. Miyazawa, and T. Usuki, Jpn. J. Appl. Phys., Part 1 43, 2110 (2004).

${ }^{17}$ S. Adachi, H. Sasakura, N. Yatsu, R. Kaji, and S. Muto, Appl. Phys. Lett. 91, 161910 (2007).

${ }^{18}$ A. W. Overhauser, Phys. Rev. 92, 411 (1953).

${ }^{19}$ Optical Orientation, Modern Problems in Condensed Matter Sciences, edited by F. Meier and B. Zakharchenya (North-Holland, New York, 1984) Vol. 8, Chaps. 2 and 5.

${ }^{20}$ A. Abragam, The Principles of Nuclear Magnetism (Clarendon, Oxford, 1961), Chap. 6.

${ }^{21}$ We assume that $f$ for the two groups are constant because in-plane distribution of excitation power around mesa structure $(\sim 150 \mathrm{~nm})$ is less than $\sim 3 \%$.

${ }^{22}$ P. Raghavan, At. Data Nucl. Data Tables 42, 189 (1989). 